\title{
Temperature dependence of the frequencies and effective damping parameters of ferrimagnetic resonance
}

\author{
F. Schlickeiser, ${ }^{1, *}$ U. Atxitia, ${ }^{2,3}$ S. Wienholdt, ${ }^{1}$ D. Hinzke, ${ }^{1}$ O. Chubykalo-Fesenko, ${ }^{3}$ and U. Nowak ${ }^{1}$ \\ ${ }^{1}$ Fachbereich Physik, Universität Konstanz, D-78457 Konstanz, Germany \\ ${ }^{2}$ Department of Physics, University of York, Heslington, York YO10 5DD United Kingdom \\ ${ }^{3}$ Instituto de Ciencia de Materiales de Madrid, CSIC, Cantoblanco, 28049 Madrid, Spain
}

(Received 3 September 2012; published 17 December 2012)

\begin{abstract}
Recent experiments on all-optical switching in GdFeCo and CoGd have raised the question about the importance of the angular momentum or the magnetization compensation point for ultrafast magnetization dynamics. We investigate the dynamics of ferrimagnets by means of computer simulations as well as analytically. The results from atomistic modeling are explained by a theory based on the two-sublattice Landau-Lifshitz-Bloch equation. Similarly to the experimental results and unlike predictions based on the macroscopic Landau-Lifshitz equation, we find an increase in the effective damping at temperatures approaching the Curie temperature. Further results for the temperature dependence of the frequencies and effective damping parameters of the normal modes represent an improvement of former approximated solutions, building a better basis for comparison to recent experiments.
\end{abstract}

DOI: 10.1103/PhysRevB.86.214416

PACS number(s): 75.78.-n, 75.50.Gg

\section{INTRODUCTION}

The recent discovery of ultrafast, optomagnetic writing schemes using circularly polarized laser pulses, ${ }^{1-3}$ pure thermal excitation, ${ }^{4,5}$ or terahertz radiation ${ }^{6}$ focuses much attention on the understanding of antiferromagnetic and ferrimagnetic materials since all these effects have been found only for materials with at least two sublattices. Switching with circularly polarized light or just with the heat pulse has been restricted to ferrimagnets with a rare-earth component, as, e.g., $\mathrm{GdFeCo}^{1}$ or $\mathrm{TbCo}^{7}$ The reason for this restriction is not fully understood, though it has been speculated that the peculiarities of the dynamics of a ferrimagnet across the angular momentum compensation temperature, where the effective damping and the frequency of the normal modes are predicted to increase rapidly ${ }^{8}$ plays a crucial role.

In general, ferrimagnetic materials with two sublattices show two characteristic damped precession motions of the total magnetization around an external field $\mathbf{H}^{0}$. As they can be excited experimentally by oscillating magnetic fields, they are called resonance modes. For one mode both sublattices stay antiparallel to each other. The dynamics related to this mode can be described as an effective ferromagnetic system and is called the ferromagnetic mode (FMM). The other normal mode is caused by the antiferromagnetic coupling between the two sublattices. In this so-called exchange mode (EXM), the sublattices are tilted at a characteristic angle. ${ }^{9}$ The characteristic motion of both modes is shown in Fig. 1.

The parameters that basically define the possible switching time are the frequency and the effective damping of the resonance modes of the samples. Both need to be high in order to enable fast magnetization reversal. The temperature dependence of the dynamic behavior of ferrimagnets is of special interest here since in earlier theories of ferrimagnetic resonance ${ }^{8,10-12}$ based on the two-macrospin Landau-LifshitzGilbert (LLG) equation of motion, the FMM shows a divergence of (or at least a rapid increase in) the frequency and the effective damping parameter at the angular momentum compensation temperature $T_{\mathrm{A}}$.
Recently, the temperature dependence of these resonance modes was investigated experimentally for amorphous, ferrimagnetic GdFeCo by Stanciu et al. ${ }^{13}$ and for amorphous, ferrimagnetic CoGd by Binder et al. ${ }^{14}$ In both experiments it was shown that both the frequency and the effective damping parameter of the FMM increase significantly, approaching the angular momentum compensation point $T_{\mathrm{A}}$. Besides this partial coincidence with the analytical prediction for the frequency of the FMM, the experimental findings in Ref. 13 also feature some disagreement with earlier theories. The common approximate solution by Wangsness ${ }^{8}$ predicts that the frequency will go to 0 at the magnetization compensation point $T_{\mathrm{M}}$, while in the experiment its value remains finite, not even with a minimum. For the experimentally observed effective damping parameter the disagreement with earlier theories is even more pronounced. Unlike the theoretical predictions in the experiment the effective damping is observed to increase significantly, approaching the Curie temperature $T_{\mathrm{C}}$.

In this work, we present a more general analytical solution based on the Landau-Lifshitz-Bloch ${ }^{15}$ (LLB) equation of motion for the temperature dependence of the frequency and effective damping parameters of both modes and compare them with our numerical findings from atomistic spin-model simulations. We show that the assumption of a temperatureindependent sublattice damping parameter, confirmed experimentally for a wide range of temperatures ${ }^{16}$ far below $T_{\mathrm{C}}$, does not hold in the high-temperature regime close to $T_{\mathrm{C}}$, and we present the derivation of new temperature-dependent damping parameters. Additionally, we recall the invalidity of some common approximate solutions at the compensation points and show the influence of the strength of a magnetocrystalline anisotropy on the properties of the resonance modes, enabling an understanding of the experimental findings.

\section{NUMERICAL METHODS}

\section{A. Model}

Our numerical results are based on a spin model where we consider classical spins $\mathbf{S}_{v, \kappa}=\boldsymbol{\mu}_{v, \kappa} / \mu_{v, \kappa}$ on two different 
(a)
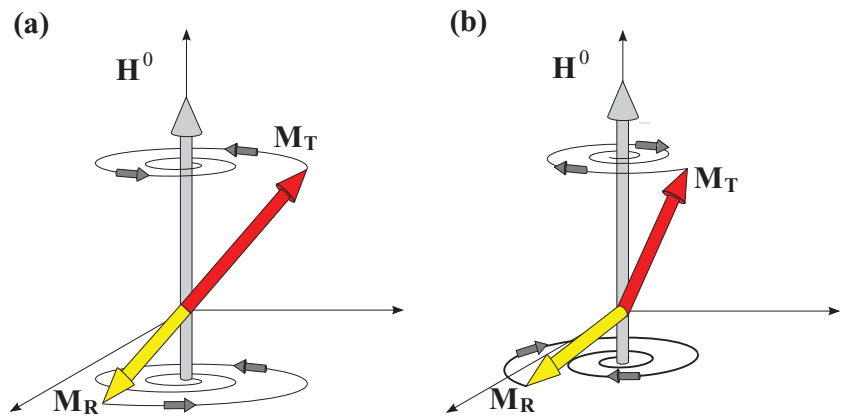

FIG. 1. (Color online) Schematic of the two resonance modes in ferrimagnets. (a) For the ferromagnetic mode, the sublattices remain antiparallel; (b) for the exchange mode, the sublattices are tilted at a characteristic angle.

sublattices. Here, $v, \kappa=\mathrm{T}, \mathrm{R}$ with $\kappa \neq v$ represents either the rare-earth-metal $(\mathrm{R})$ or the transition-metal $(\mathrm{T})$ sublattice, and $\mu_{v}$ is the atomic magnetic moment with $\mu_{\mathrm{R}} / \mu_{\mathrm{T}}=2$. The position of the spins, which are localized regularly on the two intertwined sublattices of a simple cubic lattice, is chosen such that nearest neighbors (nn's) always belong to the other sublattice respectively. The contribution to the Hamiltonian from one single spin is

$$
\begin{aligned}
\mathcal{H}_{\nu}^{i}= & -\frac{1}{2} \sum_{j \in \mathrm{nnn}} J_{\nu} \mathbf{S}_{\nu}^{i} \mathbf{S}_{\nu}^{j}-\frac{1}{2} \sum_{j \in \mathrm{nn}} J_{\nu \kappa} \mathbf{S}_{\nu}^{i} \mathbf{S}_{\kappa}^{j} \\
& -d_{\nu}^{z}\left(S_{v}^{z, i}\right)^{2}-\mu_{\nu} \mathbf{H}^{0} \mathbf{S}_{\nu}^{i} .
\end{aligned}
$$

Here, the first sum represents the ferromagnetic coupling between spins in the same sublattice [next-nearest-neighbor (nnn) interaction], while the second sum represents the antiferromagnetic interaction between spins in different sublattices (nn). Besides the exchange interaction with reduced values $J_{\mathrm{R}} / J_{\mathrm{T}}=0.2$ and $J_{\mathrm{RT}} / J_{\mathrm{T}}=-0.1$, we consider also a magnetocrystalline anisotropy in the $z$ direction with the anisotropy constant $d_{v}^{z}$ (which is varied) as well as the Zeeman energy from an external magnetic field $\mathbf{H}^{0}$.

Our numerical results for $N=32^{3}$ spins are generated by solving the stochastic Landau-Lifshitz ${ }^{17}$ equation via Heun's method. The equation itself and the method used are described in detail in Ref. 18. For the gyromagnetic ratios we use $\gamma_{\mathrm{R}} / \gamma_{\mathrm{T}}=0.75$, and as the microscopic damping constant, describing the coupling of the spin system to the heat bath, we use $\lambda=0.01$ for the nonanisotropic case and $\lambda=0.001$ for finite anisotropy. The heat bath is provided by the electronic degrees of freedom as well as by the lattice and it defines the temperature of our simulations in the canonical ensemble.

\section{B. Equilibrium magnetizations and transverse relaxation}

The sublattice equilibrium magnetizations are calculated as the spatial and time average of the (easy axis) $z$ component of the magnetic moments,

$$
M_{v}^{\mathrm{e}}=\frac{\mu_{v}}{N_{\nu} a^{3}} \cdot\left\langle\sum_{i=1}^{N_{v}} S_{v}^{z, i}\right\rangle,
$$

with $v=\mathrm{R}, \mathrm{T}$, and $N_{v}$ defining the number of unit cells with volume $a^{3}$ in the system. The temperature dependence

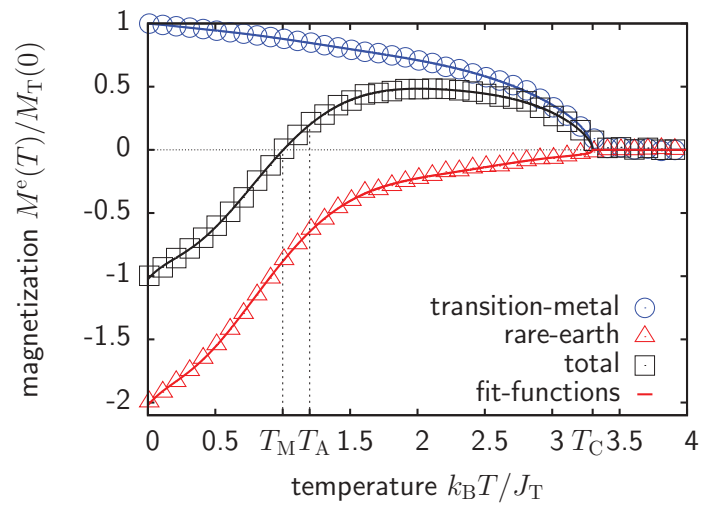

FIG. 2. (Color online) Temperature dependence of the equilibrium magnetization of the sublattices. At the magnetization compensation point, $T_{\mathrm{M}}$, the sublattice magnetizations cancel each other, while at the angular momentum compensation point, $T_{\mathrm{A}}$, the angular momenta of both sublattices are equal. Above the critical point $T_{\mathrm{C}}$ the system is paramagnetic.

of the resulting sublattice and total magnetizations, basically determined by the respective exchange constants, are shown in Fig. 2. We note that, due to their coupling, the two sublattices have the same critical temperature. In addition to this Curie temperature, ferrimagnets may have two other characteristic temperatures relevant to their magnetic behavior. At the magnetization compensation point $T_{\mathrm{M}}$ the sublattice magnetizations cancel each other, so that the total magnetization $M_{\text {total }}^{\mathrm{e}}=M_{\mathrm{R}}^{\mathrm{e}}+M_{\mathrm{T}}^{\mathrm{e}}$ is 0 . This point can exist in ferrimagnets where the sublattice with the larger zero-temperature magnetization has a weaker ferromagnetic coupling, so that the magnetization decays more rapidly compared to the other sublattice. Additionally, if both sublattices have different gyromagnetic ratios there is an angular momentum compensation point $T_{\mathrm{A}}$ where the angular momenta of both sublattices are equal, $M_{\mathrm{T}}^{\mathrm{e}} / \gamma_{\mathrm{T}}=M_{\mathrm{R}}^{\mathrm{e}} / \gamma_{\mathrm{R}}$. The solid lines in Fig. 2 correspond to fit functions, which are important when numerical simulations are compared with analytical predictions in the following analysis. They are obtained via a polynomial fitting procedure which includes the mean-field critical behavior close to the Curie temperature.

In our simulations the excitation of either the FMM or the EXM is done separately, by first loading a multispin configuration from equilibrium calculations for that temperature (Fig. 2) and then tilting the spin system with respect to an external magnetic field $\mathbf{H}^{0}$. While the FMM is excited by tilting the total system by $30^{\circ}$ with respect to the external magnetic field, for the EXM the angles between each sublattice and the external magnetic field are varied separately, since the characteristic angle between the sublattices (Fig. 1) is temperature dependent. ${ }^{11}$ In this work the external magnetic field is always parallel to the $z$ axis. Therefore the time development of the $x$ and $y$ components of the magnetization for both modes follows a damped precession motion with

$$
M_{x, y}(t) \propto \exp (-b t) \cdot \cos (\omega t+\phi),
$$

where $b$ represents the damping rate, $\omega$ is the frequency, and $\phi$ corresponds to a phase shift. The different frequencies and 
damping rates for either the FMM or the EXM have been obtained by fitting directly to the resulting time development of the $x$ and $y$ components of the magnetization. Alternatively, we tried to obtain the parameters above from a Fourier transformation of the time-dependent magnetization data. However, these results turned out to be less accurate, probably due to the fact that our simulations are very time-consuming, and consequently, the number of oscillations is not sufficient for an analysis via Fourier transformation.

\section{PROPERTIES OF THE NORMAL MODES}

\section{A. Transverse relaxation within the Landau-Lifshitz (LL) equation}

Earlier analytical calculations of the normal modes have been based on two coupled nonthermal equations of motion for the macroscopic magnetizations of sublattices using certain approximations. $^{8,10-12}$ Here we want to go beyond these restrictions, first avoiding approximations and in the next section including thermal effects via the LLB equation. We will see that the use of the LLB equation will only affect the temperature dependence of the damping.

Considering, in a two-sublattice micromagnetic LL equation, only intersublattice exchange, the Zeeman energy, and the magnetorystalline anisotropy, the effective fields of both sublattices are given by $\mathbf{H}_{v}^{\text {eff }}=\mathbf{H}^{0}+\mathbf{H}_{v}^{\text {ex }}+\mathbf{H}_{v}^{\text {an }}$. With the magnetic field and the magnetocrystalline anisotropy parallel to the $z$ axis, the effective field contributions become $\mathbf{H}^{0}=$ $H^{0} \mathbf{e}_{z}, \mathbf{H}_{v}^{\mathrm{ex}}=-A \mathbf{M}_{\kappa}$, and $\mathbf{H}_{v}^{\mathrm{an}}= \pm 2 D_{v}^{z} \mathbf{M}_{v}^{\mathrm{e}, z} \mathbf{e}_{z}$. Here, $A$ represents the interlattice micromagnetic exchange stiffness and $D_{v}^{z}$ is the micromagnetic anisotropy constant. By comparing these expressions with the corresponding effective fields $\mathbf{H}_{v}^{i}=$ $-1 / \mu_{v} \cdot \partial \mathcal{H}_{v}^{i} / \partial \mathbf{S}_{v}^{i}$ for the spin model [Eq. (1)], we obtain the relations between atomistic and micromagnetic parameters $A=\eta J_{\mathrm{RT}} / \mu_{\mathrm{T}} \mu_{\mathrm{R}}$ and $D_{v}^{z}=d_{\nu}^{z} / \mu_{\nu}^{2}$, with $\nu, \kappa=\mathrm{T}, \mathrm{R}$ and $\kappa \neq v$ as well as $\eta$ representing the number of nn's. In what follows we use unit vectors $\mathbf{n}_{v}=\mathbf{M}_{v} / M_{v}^{\mathrm{e}}$.

The equations of motion for the two sublattices read

$$
\begin{aligned}
\frac{\dot{\mathbf{n}_{v}}}{\gamma_{v}}= & -\left(\mathbf{n}_{v} \times \mathbf{H}_{v}^{\prime}\right)-\alpha_{v}\left[\mathbf{n}_{v} \times\left(\mathbf{n}_{v} \times \mathbf{H}_{v}^{\prime}\right)\right] \\
& +A M_{\kappa}^{\mathrm{e}}\left\{\left(\mathbf{n}_{v} \times \mathbf{n}_{\kappa}\right)+\alpha_{v} A M_{\kappa}^{\mathrm{e}}\left[\mathbf{n}_{v} \times\left(\mathbf{n}_{v} \times \mathbf{n}_{\kappa}\right)\right]\right\},
\end{aligned}
$$

where $M_{\kappa}^{\mathrm{e}}$ represents the equilibrium magnetization of the respective other sublattice, $\mathbf{H}_{v}^{\prime}=\mathbf{H}^{0}+\mathbf{H}_{v}^{\mathrm{an}}, \gamma_{v}$ are the atomistic gyromagnetic ratios, and $\alpha_{v}$ are the damping constants.

Close to equilibrium, with $\mathbf{n}_{\mathrm{T}}=n_{\mathrm{T}}\left(n_{\mathrm{T}}^{x}, n_{\mathrm{T}}^{y}, 1\right)$ and $\mathbf{n}_{\mathrm{R}}=$ $n_{\mathrm{R}}\left(n_{\mathrm{R}}^{x}, n_{\mathrm{R}}^{y},-1\right)$, we can consider $\partial_{t} n_{\mathrm{T}(\mathrm{R})}^{z}=0, M_{v}^{z} \approx M_{v}^{\mathrm{e}, z}$, and neglect second-order terms, leading to

$$
\begin{aligned}
\frac{\dot{n}_{v}^{x}}{\gamma_{\nu}}= & \left(-n_{v}^{y} \mp \alpha_{v} n_{v}^{x}\right)\left(H^{0} \pm 2 D_{v}^{z} M_{v}^{\mathrm{e}}\right) \\
& -A M_{\kappa}^{\mathrm{e}}\left(\alpha_{v}\left(n_{v}^{x}+n_{\kappa}^{x}\right) \mp\left(n_{v}^{y}+n_{\kappa}^{y}\right)\right)
\end{aligned}
$$

and

$$
\begin{aligned}
\frac{\dot{n}_{v}^{y}}{\gamma_{v}} & =\left(n_{v}^{x} \mp \alpha_{v} n_{v}^{y}\right)\left(H^{0} \pm 2 D_{v}^{z} M_{v}^{\mathrm{e}}\right) \\
& \pm A M_{\kappa}^{\mathrm{e}}\left(\left(n_{v}^{x}+n_{\kappa}^{x}\right)-\alpha_{v}\left(n_{v}^{y}+n_{\kappa}^{y}\right)\right) .
\end{aligned}
$$

Here, the upper algebraic sign is for the transition metal, while the lower one is for the rare-earth metal. By transforming into the variables of the rotating system $n_{v}^{+}=n_{v}^{x}+i n_{v}^{y}$ and $n_{v}^{-}-=n_{v}^{x}-i n_{v}^{y}$ and assuming an exponential solution $n_{v}^{ \pm}=$ $n_{v}^{0 \pm} \exp (i \tilde{\omega} t)$, we obtain

$$
\begin{aligned}
( \pm \tilde{\omega} & \left.-\gamma_{\mathrm{T}}\left(H^{0}+2 D_{\mathrm{T}}^{z} M_{\mathrm{T}}^{\mathrm{e}}+A M_{\mathrm{R}}^{\mathrm{e}}\right)\left(1 \pm i \alpha_{\mathrm{T}}\right)\right) n_{\mathrm{T}}^{ \pm} \\
& -\gamma_{\mathrm{T}} A M_{\mathrm{R}}^{\mathrm{e}}\left(1 \pm i \alpha_{\mathrm{T}}\right) n_{\mathrm{R}}^{ \pm}=0, \\
( \pm \tilde{\omega} & \left.-\gamma_{\mathrm{R}}\left(H^{0}-2 D_{\mathrm{R}}^{z} M_{\mathrm{R}}^{\mathrm{e}}-A M_{\mathrm{T}}^{\mathrm{e}}\right)\left(1 \mp i \alpha_{\mathrm{R}}\right)\right) n_{\mathrm{R}}^{ \pm} \\
& +\gamma_{\mathrm{R}} A M_{\mathrm{T}}^{\mathrm{e}}\left(1 \mp i \alpha_{\mathrm{R}}\right) n_{\mathrm{T}}^{ \pm}=0 .
\end{aligned}
$$

The solution for the frequencies corresponds to the real part of the two independent solutions for the FMM and EXM, respectively, and the damping rate is given by the imaginary part. The effective damping parameter is then given by the ratio of damping rate to frequency, ${ }^{11} \alpha_{\mathrm{eff}}=b_{\mathrm{fm}, \mathrm{ex}} / \omega_{\mathrm{fm}, \mathrm{ex}}$. Due to their length, we do not write down these equations, but we will use the full solution later for comparison with numerical data and an improved analytical approach. Note, however, that the effective damping is the same for both modes if the sublattice damping parameters $\alpha_{v}$ are assumed to be equal.

Based on this approach several approximated solutions for the frequencies and effective damping parameter have been derived in the past. However, the most common solutions for the frequencies, by Wangsness ${ }^{10}$ for the FMM,

$$
\omega_{\mathrm{FMM}}=\frac{\gamma_{\mathrm{T}} \gamma_{\mathrm{R}}\left(M_{\mathrm{T}}^{\mathrm{e}}-M_{\mathrm{R}}^{\mathrm{e}}\right)}{\left(\gamma_{\mathrm{R}} M_{\mathrm{T}}^{\mathrm{e}}-\gamma_{\mathrm{T}} M_{\mathrm{R}}^{\mathrm{e}}\right)} H^{0},
$$

and by Kaplan and $\mathrm{Kittel}^{9}$ for the EXM,

$$
\omega_{\mathrm{EXM}}=A\left(\gamma_{\mathrm{T}} M_{\mathrm{R}}^{\mathrm{e}}-\gamma_{\mathrm{R}} M_{\mathrm{T}}^{\mathrm{e}}\right),
$$

make use of two main approximations: first, they neglect the influence of damping and anisotropy completely; and second, they include the assumptions $A M_{v}^{\mathrm{e}} \gg H^{0}$, which fails close to the Curie temperature $T_{\mathrm{C}} ; A\left(M_{\mathrm{T}}^{\mathrm{e}}-M_{\mathrm{R}}^{\mathrm{e}}\right) \gg$ $H^{0}$, which fails close to the magnetization compensation point $T_{\mathrm{M}}$; and $A\left(\gamma_{\mathrm{T}} M_{\mathrm{R}}^{\mathrm{e}}-\gamma_{\mathrm{R}} M_{\mathrm{T}}^{\mathrm{e}}\right) \gg H^{0}$, which fails close to the angular momentum compensation point. Thus, these approximations predict an erroneous behavior at and close to these characteristic temperatures. Similar approximations in calculations of the effective damping parameter ${ }^{11}$ and the solution for the frequency of the finite-anisotropy case by Walker ${ }^{19}$ fail here correspondingly. Note that also the solution of the effective damping parameter, ${ }^{11}$

$$
\alpha_{\mathrm{eff}}=\frac{M_{\mathrm{R}}^{\mathrm{e}} \gamma_{\mathrm{T}} \alpha_{\mathrm{R}}+\gamma_{\mathrm{R}} \alpha_{\mathrm{T}} M_{\mathrm{T}}^{\mathrm{e}}}{M_{\mathrm{R}}^{\mathrm{e}} \gamma_{\mathrm{T}}-M_{\mathrm{T}}^{\mathrm{e}} \gamma_{\mathrm{R}}},
$$

predicts a divergence at $T_{\mathrm{A}}$ and therefore zero switching time. As we will show in the following, in the full analytical solution neither the frequencies nor the effective damping parameters diverge at $T_{\mathrm{A}}$. Instead, we find only characteristic maxima at or close to the angular momentum compensation point.

\section{B. Temperature-dependent transverse relaxation within the LLB theory for ferrimagnets}

The recently published derivation of the LLB equation for two-component systems in Ref. 21 explicitly refers to a disordered ferrimagnet. Since for this work we consider an ordered ferrimagnet, we briefly repeat the derivation and 
present the formula for the ordered case in the explicit form. In the following we derive the macroscopic equation for the thermally averaged spin polarization $\mathbf{m}_{v}=\left\langle S_{i}^{v}\right\rangle$ in each sublattice $v=\mathrm{T}, \mathrm{R}$, following the theory of the LLB equation for ferromagnets. ${ }^{15}$ The derivation uses a mean-field approximation (MFA). Since in the present article we are not interested in longitudinal motion, observed on the time scale of $100 \mathrm{fs}$ to $1 \mathrm{ps}$, we focus our attention on the LLB equation with transverse motion only. Additionally, the longitudinal normal modes are decoupled from the transverse ones, which allows for their separate consideration. Such an approximation leads to the following sets of coupled LLB equations: ${ }^{15}$

$$
\dot{\mathbf{m}}_{v}=\gamma_{v}\left[\mathbf{m}_{v} \times \mathbf{H}_{v}^{\mathrm{MFA}}\right]-\Gamma_{\perp}^{v} \frac{\left[\mathbf{m}_{v} \times\left[\mathbf{m}_{v} \times \mathbf{m}_{v, 0}\right]\right]}{m_{v}^{2}}
$$

with

$$
\mathbf{m}_{v, 0}=B\left(\xi_{v, 0}\right) \frac{\boldsymbol{\xi}_{v, 0}}{\xi_{v, 0}}, \quad \boldsymbol{\xi}_{v, 0} \equiv \frac{\mu_{v} \mathbf{H}_{v}^{\mathrm{MFA}}}{k_{\mathrm{B}} T},
$$

where $\mathbf{H}_{v}^{\mathrm{MFA}}$ is the average mean field acting on the spin, and the relaxation rates are given by

$$
\Gamma_{\perp}^{v}=\frac{\gamma_{\nu} \lambda_{\nu} k_{\mathrm{B}} T}{\mu_{\nu}}\left(\frac{\xi_{v, 0}}{B\left(\xi_{v, 0}\right)}-1\right),
$$

where $B(\xi)=\operatorname{coth}(\xi)-1 / \xi$ is the Langevin function. In Eq. (11) the first term describes the magnetization precession and the second term the transverse relaxation. The next step is to use the MFA in Eqs. (12). The MFA expression for fields in a ferrimagnet are well known; see also recent results for FeCoGd. ${ }^{20}$ Defining $\mathbf{H}_{\text {eff. } \mathrm{T}(\mathrm{R})}^{\prime}=\mathbf{H}+\mathbf{H}_{A, \mathrm{~T}(\mathrm{R})}$ as the sum of the external and anisotropy fields in each sublattice, we can write the average molecular field acting at each sublattice spin as

$$
\begin{gathered}
\mu_{\mathrm{R}} \mathbf{H}_{\mathrm{R}}^{\mathrm{MFA}}=\mu_{\mathrm{R}} \mathbf{H}_{\mathrm{eff}, \mathrm{R}}^{\prime}+J_{0, \mathrm{R}} \mathbf{m}_{\mathrm{R}}+J_{0, \mathrm{TR}} \mathbf{m}_{\mathrm{T}}, \\
\mu_{\mathrm{T}} \mathbf{H}_{\mathrm{T}}^{\mathrm{MFA}}=\mu_{\mathrm{T}} \mathbf{H}_{\mathrm{eff}, \mathrm{T}}^{\prime}+J_{0, \mathrm{~T}} \mathbf{m}_{\mathrm{T}}+J_{0, \mathrm{TR}} \mathbf{m}_{\mathrm{R}},
\end{gathered}
$$

where $J_{0, \mathrm{~T}}=\eta J_{\mathrm{T}}, \eta$ is the number of nn's of transition-metal type for the transition-metal spin, and $J_{0, \mathrm{TR}}$ and $J_{0, \mathrm{R}}$ have similar definitions. The minimum condition for the free energy, $\partial \mathcal{F} / \partial \mathbf{m}_{\mathrm{R}}=0$ and $\partial \mathcal{F} / \partial \mathbf{m}_{\mathrm{T}}=0$, leads to the coupled CurieWeiss equations,

$$
\mathbf{m}_{\mathrm{R}}=B\left(\xi_{\mathrm{R}, 0}\right) \frac{\boldsymbol{\xi}_{\mathrm{R}, 0}}{\xi_{\mathrm{R}, 0}}, \quad \mathbf{m}_{\mathrm{T}, 0}=B\left(\xi_{\mathrm{T}, 0}\right) \frac{\boldsymbol{\xi}_{\mathrm{T}, 0}}{\xi_{\mathrm{T}, 0}},
$$

the self-consistent solutions of which are the equilibrium magnetization of each sublattice.

We treat the most general case where the continuous approximation in each sublattice can be used. In order to simplify the problem we decompose the magnetization vector $\mathbf{m}_{v}$ into two components, $\mathbf{m}_{v}=\boldsymbol{\Pi}_{v}+\boldsymbol{\tau}_{v}$, where $\boldsymbol{\Pi}_{v}$ is perpendicular to $\mathbf{m}_{\kappa}$, so that it can be expressed as $\boldsymbol{\Pi}_{v}=$ $-\left[\mathbf{m}_{\kappa} \times\left[\mathbf{m}_{\kappa} \times \mathbf{m}_{v}\right]\right] / m_{\kappa}^{2}$, and $\boldsymbol{\tau}_{v}$ is parallel to $\mathbf{m}_{\kappa}$, so that it can be expressed as $\boldsymbol{\tau}_{v}=\mathbf{m}_{\kappa}\left(\mathbf{m}_{v} \cdot \mathbf{m}_{\kappa}\right) / m_{\kappa}^{2}$, where $\kappa \neq v$.

Similarly, the MFA exchange field $\mathbf{H}_{\mathrm{EX}, v}^{\mathrm{MFA}}$ in Eqs. (15) and (14) can be written as the sum of the exchange field parallel and perpendicular to magnetization of the sublattice $v$,

$$
\mathbf{H}_{\mathrm{EX}, v}^{\mathrm{MFA}}=\mathbf{H}_{\mathrm{EX}, v}^{\|}+\mathbf{H}_{\mathrm{EX}, v}^{\perp}=\frac{\widetilde{J}_{0, v}}{\mu_{v}} \mathbf{m}_{v}+\frac{J_{0, v \kappa}}{\mu_{v}} \boldsymbol{\Pi}_{\kappa},
$$

where we have defined a new function, $\widetilde{J}_{0, v}\left(\mathbf{m}_{\kappa}, \mathbf{m}_{v}\right)$, as $\widetilde{J}_{0, v}=$ $J_{0, v}+J_{0, \nu \kappa}\left(\mathbf{m}_{v} \cdot \mathbf{m}_{\kappa}\right) / m_{\kappa}^{2}$. Note that $\widetilde{J}_{0, v}$ is not a constant but a function of both sublattices' magnetizations.

In the following, we consider the case where the transverse contribution in the exchange field is small in comparison to the longitudinal one, $\left|\mathbf{H}_{\mathrm{EX}, v}^{\|}\right| \gg\left|\mathbf{H}_{\mathrm{EX}, v}^{\perp}\right|$, i.e., where the noncollinearities between sublattices are small. Finally, $\mathbf{H}_{v}^{\mathrm{MFA}} \simeq$ $\mathbf{H}_{\mathrm{EX}, v}^{\|}+\mathbf{H}_{\mathrm{eff}, v}^{\prime \prime}$, where $\mathbf{H}_{\mathrm{eff}, v}^{\prime \prime}=\mathbf{H}+\mathbf{H}_{A, \text { eff }, v}+\mathbf{H}_{\mathrm{EX}, v}^{\perp}$. We now expand $\mathbf{m}_{v, 0}$ up to the first order in $\mathbf{H}_{\text {eff, }}^{\prime \prime}$, under the assumption $\left|\mathbf{H}_{\mathrm{EX}, v}^{\|}\right| \gg\left|\mathbf{H}_{\mathrm{eff}, v}^{\prime \prime}\right|$. From Eqs. (16) the value of $\mathbf{m}_{v, 0}$ in the above conditions ${ }^{21}$ can be substituted into Eq. (11), leading to the following equation of motion:

$$
\dot{\mathbf{m}}_{v}=\gamma_{v}\left[\mathbf{m}_{v} \times \mathbf{H}_{\mathrm{eff}, v}^{\prime \prime}\right]-\Gamma_{\perp}^{v} \frac{B_{v} \mu_{v}}{m_{v} \tilde{J}_{0, v}} \frac{\left[\mathbf{m}_{v} \times\left[\mathbf{m}_{v} \times \mathbf{H}_{\mathrm{eff}, v}^{\prime \prime}\right]\right]}{m_{v}^{2}} .
$$

In the same approximation we have $B_{v} \simeq m_{v}, B\left(\xi_{0, v}\right) / \xi_{0, v} \simeq$ $\left(k_{\mathrm{B}} T\right) / \tilde{J}_{0, \nu}$, and, finally,

$$
\Gamma_{\perp}^{\nu}=\frac{\gamma_{\nu} \alpha_{\perp}^{v} k_{\mathrm{B}} T}{\mu_{v}}\left(\frac{\xi_{0, v}}{B\left(\xi_{0, v}\right)}-1\right) \simeq \gamma_{\nu} \alpha_{\perp}^{\nu}\left(\frac{\tilde{J}_{0, v} m_{v}}{\mu_{\nu} B_{v}}\right),
$$

where $\alpha_{\perp}^{\nu}=\lambda_{\nu}\left(1-\frac{k_{B} T}{\widetilde{J}_{0, \nu}}\right)$. Hence the final form of the LLB equation is

$$
\dot{\mathbf{m}}_{v}=\gamma_{v}\left[\mathbf{m}_{v} \times \mathbf{H}_{\mathrm{eff}, v}^{\prime \prime}\right]-\gamma_{\nu} \alpha_{\perp}^{v} \frac{\left[\mathbf{m}_{v} \times\left[\mathbf{m}_{v} \times \mathbf{H}_{\mathrm{eff}, v}^{\prime \prime}\right]\right]}{m_{v}^{2}} .
$$

The temperature dependence of the damping parameters is obtained in the first order in deviations of magnetization from their equilibrium value. Note that in Eq. (17) all the terms are of the first order in the parameter $H_{\mathrm{eff}, v}^{\prime \prime} / H_{\mathrm{EX}, v}^{\|}$so that the damping parameters should be evaluated in the zero order in this parameter. As a result, the effective damping parameter depends on the temperature $T$ via the equilibrium magnetization values as

$$
\widetilde{J}_{0, v} \simeq \frac{J_{0, \nu} m_{v}^{\mathrm{e}}-J_{0, \nu \kappa} m_{\kappa}^{\mathrm{e}}}{m_{v}^{\mathrm{e}}}
$$

Note also that the field $\mathbf{H}_{\text {eff }}^{\prime \prime}$ could be substituted in the precession and the transverse damping terms with $\mathbf{H}_{\text {eff }}$ (including the exchange field coming from the opposite sublattice), since the action of the component of this field parallel to the magnetization $\mathbf{m}_{v}$ is 0 . Note also that Eq. (19) does not have exactly the LL form due to the presence of the $m_{v}^{2}$ term in the denominator. The difference between the LL and the LLB damping is discussed for the ferromagnetic case in Refs. 22 and 23.

For a comparision with the results in Sec. III A, Eq. (19) can be written in terms of the variable $\mathbf{n}=\mathbf{m} / \mathrm{m}^{e}$. After renormalizing the equation and linearizing it close to equilibrium at a given temperature, one gets a similar result as for the LL equation [Eqs. (5) and (6)] but with temperature-dependent parameters,

$$
\tilde{\alpha}_{\perp}^{\mathrm{T}}(T)=\frac{\alpha_{\mathrm{T}}}{m_{\mathrm{T}}^{e}(T)}\left(1-\frac{m_{\mathrm{T}}^{\mathrm{e}}(T) k_{\mathrm{B}} T}{J_{0, \mathrm{~T}} m_{\mathrm{T}}^{\mathrm{e}}(T)-J_{0, \mathrm{TR}} m_{\mathrm{R}}^{\mathrm{e}}(T)}\right),
$$


and

$$
\tilde{\alpha}_{\perp}^{\mathrm{R}}(T)=\frac{\alpha_{\mathrm{R}}}{m_{\mathrm{R}}^{e}(T)}\left(1-\frac{m_{\mathrm{R}}^{e}(T) k_{\mathrm{B}} T}{J_{0, \mathrm{R}} m_{\mathrm{R}}^{\mathrm{e}}(T)-J_{0, \mathrm{R} T} m_{\mathrm{T}}^{\mathrm{e}}(T)}\right),
$$

where the parameters $\lambda_{\mathrm{R}}, \lambda_{\mathrm{T}}$ were substituted by $\alpha_{\mathrm{R}}, \alpha_{\mathrm{T}}$ to comply with the standard notations of the micromagnetic equation. Here, similarly to the procedure described in Ref. 20, we have renormalized the exchange parameters within the MFA. The replacement of Eqs. (21) and (22) in Eq. (7) leads to an increase in the effective damping parameters for both modes at high temperatures, which agrees with the numerical findings. It is that combination of equations that we call the analytical solution in the following.

\section{RESULTS AND DISCUSSION}

Let us start with a discussion of the zero-anisotropy case. Since in our simulations an external magnetic field $H^{0}=$ $0.02 J_{\mathrm{T}} / \mu_{\mathrm{T}}$ is constantly switched on, for zero anisotropy this magnetic field will have to change its sign at the magnetization compensation point $T_{\mathrm{M}}$ in order to avoid a switching of the whole system. This change leads to the discontinuity of the analytical solutions [Eq. (7)] shown in Fig. 3 at $T_{\mathrm{M}}$. For the frequencies [Fig. 3(a)] as well as the effective damping parameters [Fig. 3(b)] we obtain a very good agreement between analytical and numerical solutions in both modes and for the whole temperature range. We note that the value of the frequency of the FMM first tends to 0 below the magnetization compensation point $T_{\mathrm{M}}$, where it starts to increase to its

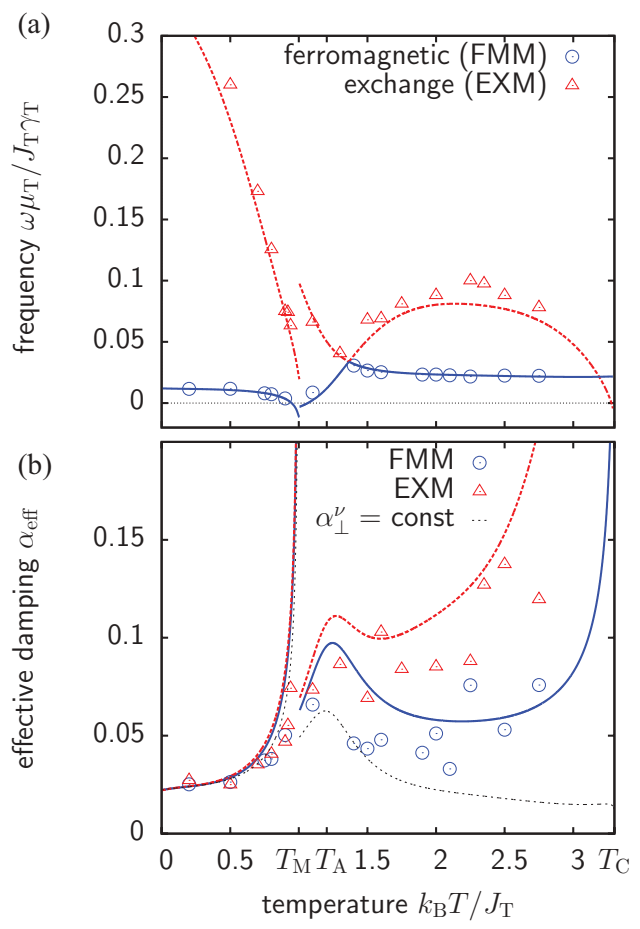

FIG. 3. (Color online) Frequencies and effective damping parameters in the zero-anisotropy case. Temperature dependence of (a) frequencies and (b) effective damping parameters $\alpha_{\text {eff }}$. Numerically obtained data points are compared with analytical solutions. The switching of the external magnetic field $\mathbf{H}^{0}$ leads to a gap in the solutions at the magnetization compensation point $T_{\mathrm{M}}$. maximum above the angular momentum compensation point $T_{\mathrm{A}}$. After decreasing with higher temperatures the value of the frequency of the FMM converges to a constant level. For the EXM the effect of changing the relative direction of the external field is stronger, since, in comparison to the approximated solution ${ }^{9}$ [Eq. (9)], the value of the EXM frequency is constantly shifted proportionally to the strength of $H^{0}$. Above the angular momentum compensation point $T_{\mathrm{A}}$ the frequencies of both modes reach the same value, where the FMM has its maximum and the EXM reaches a local minimum.

Note that there is an increase in the effective damping parameter at high temperatures that is much stronger for the EXM. Interestingly, without considering the temperature dependence of the sublattice damping parameters [Eqs. (21) and (22)] in the analytical solution [Eq. (7)] and assuming simply the microscopic damping constant $\lambda=0.01$ to describe the relaxation dynamics of the sublattice magnetizations, the effective damping parameters $\alpha_{\text {eff }}$ for both modes are equal. This solution, plotted as the dashed line in Fig. 3(b), does not coincide with our numerical data. Only considering the temperature dependence of the sublattice damping parameters, the effective damping parameters $\alpha_{\text {eff }}$ of both modes become different and describe the increase for both modes at high temperatures correctly [Fig. 3(b)]. Note also that the influence of the temperature dependence for $\tilde{\alpha}_{\perp}^{\mathrm{T}}(T)$ and $\tilde{\alpha}_{\perp}^{\mathrm{R}}(T)$ is negligible at low temperatures but becomes very important with increasing temperatures.

For the finite-anisotropy case (Fig. 4) we have used the following values as atomistic damping parameters, external

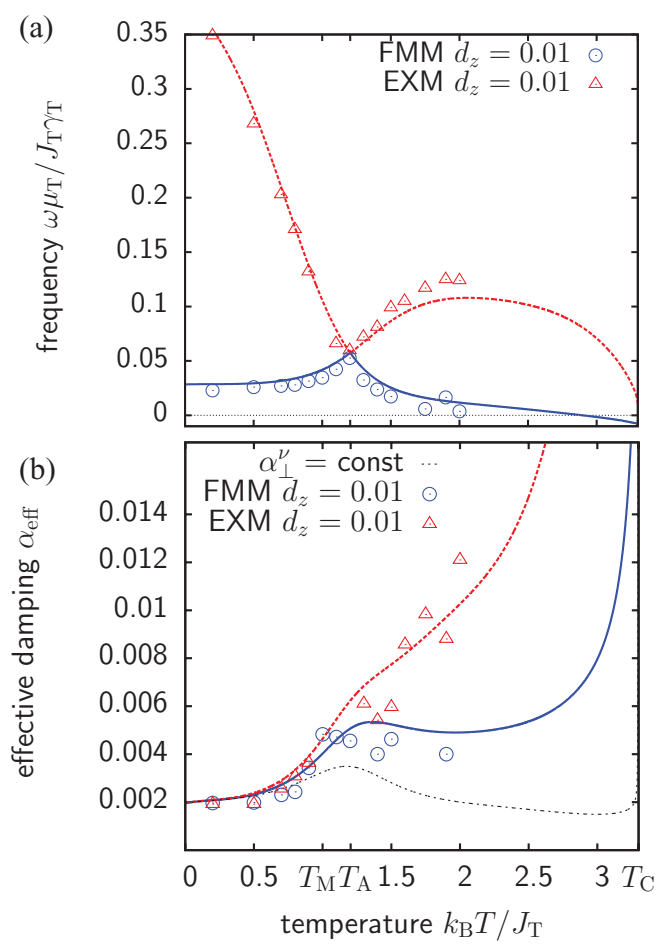

FIG. 4. (Color online) Frequencies and effective damping parameters in the finite-anisotropy case. Temperature dependence of (a) frequencies and (b) effective damping parameters $\alpha_{\text {eff }}$. Numerically obtained data points are compared with analytical solutions. 
field, and anisotropy constant: $\lambda=0.001, H^{0}=0.01, J_{\mathrm{T}} / \mu_{\mathrm{T}}$, and $d_{z}=0.01 J_{\mathrm{T}}$. Here, we have used smaller atomistic damping compared to the previous simulations, since the consideration of an anisotropy leads to an increase in the discrepancy between simulated and ideal damped modes, so that more cycles had to be fitted in order to obtain good results. With anisotropy, consequently, the resulting anisotropy field compensates the external field and avoids switching at the magnetization compensation point $T_{\mathrm{M}}$ in our simulations. Therefore we have not switched the direction of $\mathbf{H}^{0}$ in this case. For higher temperatures, however, due to thermal excitation as well as the decaying anisotropy field, the system starts switching anyway. Therefore the effective damping parameters could not be obtained for the high-temperature range.

For the frequencies [Fig. 4(a)] the consideration of a uniaxial anisotropy leads to the fact that the minimum at the magnetization compensation point vanishes. Again, above the angular momentum compensation temperature $T_{\mathrm{A}}$ we obtain a maximum for the FMM frequency and a minimum for the EXM frequency. We note that the shift of this characteristic point from $T_{\mathrm{A}}$ to higher values is proportional to the strength of the external magnetic field as well as the anisotropy.

Regarding the effective damping parameters [Fig. 4(b)] a finite anisotropy leads to a less pronounced maximum at $T_{\mathrm{A}}$. Once again, the dashed line in Fig. 4(b) corresponds to the analytical solution without consideration of the temperature dependence of the sublattice damping, leading to the equality of the effective damping parameters of both modes, not showing the increase in $\alpha_{\text {eff }}$ for higher temperatures. Besides the good agreement between numerical and analytical results when the temperature dependence of the sublattice damping is taken into account, we now also obtain a good agreement with the experimental findings of Stanciu et al. ${ }^{13}$ Thus we are able to reproduce these experimental findings qualitatively by considering a uniaxial, magnetocrystalline anisotropy as well as temperature-dependent sublattice damping parameters as derived within the framework of the LLB equation. These coinciding findings clearly demonstrate the failure of the analytical solutions based on the LL and LLG equations of motion $^{8,10-12}$ for high temperatures.

In Fig. 5 the analytical solutions [Eqs. (7), (21), and (22)] of the frequencies of both modes as well as the effective damping parameter for the FMM are shown for different strengths of the uniaxial anisotropy. Here, with $H^{0}=0.01 J_{\mathrm{T}} / \mu_{\mathrm{T}}$ and $\lambda=0.01$, we have also not switched the external field at $T_{\mathrm{M}}$. First, we note that due to the temperature dependence of the anisotropy field in the high-temperature regime, the influence of the strength of the anisotropy constant $d_{z}$ becomes smaller with increasing temperatures. This effect leads to the convergence of all sets of curves for different anisotropies with increasing temperatures up to $T_{\mathrm{C}}$, where the anisotropy fields vanish and the different curves join. Second, we see that the frequencies of both modes increase with increasing anisotropy. This effect is much stronger for the FMM. Additionally, the maximum of the frequency of the FMM as well as the minimum of the frequency of the EXM are shifted from the the angular momentum compensation point $T_{\mathrm{A}}$ towards higher temperatures with increasing anisotropy. For the effective damping parameter, with increasing anisotropy (a)

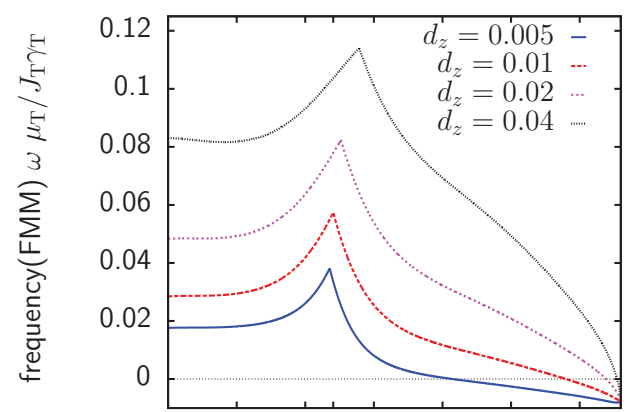

(b)

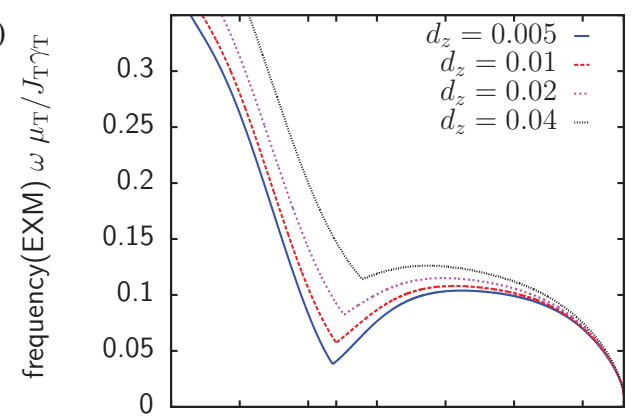

(c)

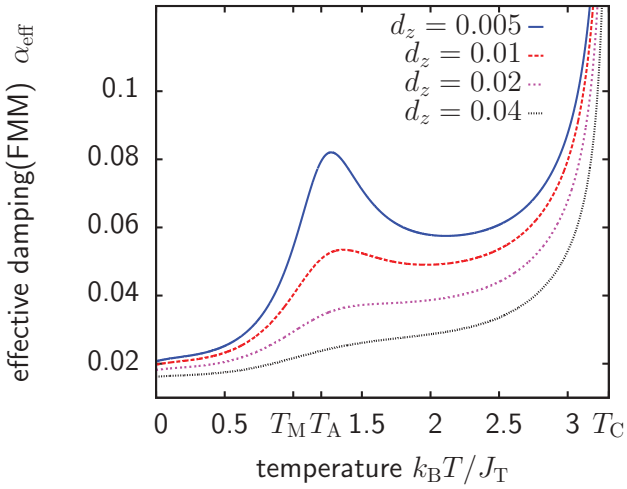

FIG. 5. (Color online) Frequencies and effective damping parameters in the finite-anisotropy case. Temperature dependence of (a) the ferromagnetic mode frequency, (b) the exchange mode frequency, and (c) the effective damping parameter $\alpha_{\text {eff }}$ of the FMM for different strengths of the magnetocrystalline anisotropy. Analytical results as explained in the text.

we obtain a decrease and a washing-out of the maximum close to $T_{\mathrm{A}}$.

\section{CONCLUSIONS}

A detailed investigation of the dynamics of ferrimagnets was performed by means of computer simulations as well as analytically. Formulas were derived for the frequencies and effective damping parameters of bot, the FMM and the EXM. We show that a correct calculation does not predict any divergence either of the effective damping parameters or of the frequencies close to the angular momentum compensation point, but only a finite maximum. Nevertheless, both the frequencies and the effective damping parameters strongly depend on the temperature, with that explaining the large variations of relaxation times in ferrimagnets, especially in optomagnetic experiments with pronounced heating effects.

Similarly to the experimental results (see Fig. 3 in Ref. 13) and unlike predictions based on the macroscopic, 
two-sublattice LLG $^{8,10-12}$ equation, we find an increase in the effective damping at a temperature approaching the Curie temperature. This stresses the importance and validity of the recently derived two-sublattice LLB equation for finite-temperature micromagnetics. The latter builds a new basis for finite-temperature micromagnetic calculations of ferrimagnets.

\section{ACKNOWLEDGMENTS}

This research received funding from the European Commission via the 7th Framework Programme grant FEMTOSPIN. The authors in Madrid also acknowledge funding by the Spanish Ministry of Science and Innovation under Grant No. FIS2010-20979-C02-02.
*Correspondence author: frank.schlickeiser@uni-konstanz.de

${ }^{1}$ C. D. Stanciu, F. Hansteen, A. V. Kimel, A. Kirilyuk, A. Tsukamoto, A. Itoh, and Th. Rasing, Phys. Rev. Lett. 99, 047601 (2007).

${ }^{2}$ A. V. Kimel, A. Kirilyuk, P. A. Usachev, R. V. Pisarev, A. M. Balbashov, and Th. Rasing, Nature 435, 655 (2005).

${ }^{3}$ K. Vahaplar, A. M. Kalashnikova, A. V. Kimel, D. Hinzke, U. Nowak, R. Chantrell, A. Tsukamoto, A. Itoh, A. Kirilyuk, and Th. Rasing, Phys. Rev. Lett. 103, 117201 (2009).

${ }^{4}$ I. Radu, K. Vahaplar, C. Stamm, T. Kachel, N. Pontius, H. A. Dürr, T. A. Ostler, J. Barker, R. F. L. Evans, R. W. Chantrell, A. Tsukamoto, A. Itoh, A. Kirilyuk, Th. Rasing, and A. V. Kimel, Nature 472, 205 (2011).

${ }^{5}$ T. A. Ostler, J. Barker, R. F. L. Evans, R. W. Chantrell, U. Atxitia, O. Chubykalo-Fesenko, S. El Moussaoui, L. Le Guyader, E. Mengotti, L. J. Heyderman, F. Nolting, A. Tsukamoto, A. Itoh, D. Afanasiev, B. A. Ivanov, A. M. Kalashnikova, K. Vahaplar, J. Mentink, A. Kirilyuk, Th. Rasing, and A. V. Kimel, Nature Commun. 3, 666 (2012).

${ }^{6}$ S. Wienholdt, D. Hinzke, and U. Nowak, Phys. Rev. Lett. 108, 247207 (2012).

${ }^{7}$ S. Alebrand, A. Hassdenteufel, D. Steil, M. Bader, A. Fischer, M. Cinchetti, and M. Aeschlimann, Phys. Status Solidi A 209, 2589 (2012).

${ }^{8}$ R. Wangsness, Phys. Rev. 91, 1085 (1953).

${ }^{9}$ J. Kaplan, and C. Kittel, J. Chem. Phys. 21, 760 (1953).

${ }^{10}$ R. Wangsness, Phys. Rev. 93, 68 (1954).
${ }^{11}$ A. G. Gurievich and G. A. Melkov, Magnetisation Oscillations and Waves (CRC Press, Boca Raton, FL, 1965).

${ }^{12}$ B. Lax and K. J. Button, Microwave Ferrites and Ferrimagnets (McGraw-Hill, New York, 1962).

${ }^{13}$ C. D. Stanciu, A. V. Kimel, F. Hansteen, A. Tsukamoto, A. Itoh, A. Kiriliyuk, and Th. Rasing, Phys. Rev. B 73, 220402 (2006).

${ }^{14} \mathrm{M}$. Binder, A. Weber, O. Mosendz, G. Woltersdorf, M. Izquierdo, I. Neudecker, J. R. Dahn, T. D. Hatchard, J.-U. Thiele, C. H. Back, and M. R. Scheinfein, Phys. Rev. B 74, 134404 (2006).

${ }^{15}$ D. A. Garanin, Phys. Rev. B 55, 3050 (1997).

${ }^{16}$ S. M. Bhagat and P. Lubitz, Phys. Rev. B 10, 179 (1974).

${ }^{17}$ L. Landau and E. Lifshitz, Phys. Z. Sowjet. 8, 153 (1935).

${ }^{18}$ U. Nowak, Handbook of Magnetism and Advanced Magnetic Materials, edited by H. Kronmüller and S. Parkin (John Wiley \& Sons, Chichester, UK, 2007), Vol. 2.

${ }^{19}$ N. Geschwind and L. R. Walker, J. Appl. Phys. 30, 163S (1959).

${ }^{20}$ T. A. Ostler, R. F. L. Evans, R. W. Chantrell, U. Atxitia, O. Chubykalo-Fesenko, I. Radu, R. Abrudan, F. Radu, A. Tsukamoto, A. Itoh, A. Kirilyuk, Th. Rasing, and A. Kimel, Phys. Rev. B 84, 024407 (2011).

${ }^{21}$ U. Atxitia, P. Nieves, and O. Chubykalo-Fesenko, Phys. Rev. B 86, 104414 (2012).

${ }^{22}$ D. A. Garanin and O. Chubykalo-Fesenko, Phys. Rev. B 70, 212409 (2004).

${ }^{23}$ O. Chubykalo-Fesenko, U. Nowak, R. W. Chantrell, and D. A. Garanin, Phys. Rev. B 74, 094436 (2006). 